\title{
Silicene passivation by few-layer graphene
}

\author{
Viktoria Ritter $^{1}$, Jakob Genser ${ }^{1}$, Daniele Nazzari ${ }^{1}$, Ole Bethge ${ }^{2}$, Emmerich Bertagnolli ${ }^{1}$, and \\ Alois Lugstein ${ }^{1 *}$
}

\footnotetext{
${ }^{1}$ Institute of Solid State Electronics, Technische Universität Wien, Gußhausstraße 25-25a, 1040 Vienna, Austria

${ }^{2}$ Infineon Technologies Austria AG, Siemensstraße 2, 9500 Villach, Austria

*alois.lugstein@tuwien.ac.at
}

Keywords: silicene, graphene, passivation, Raman spectroscopy, 2D-materials

This document is the Accepted Manuscript version of a Published Work that appeared in final form in ACS Applied Materials \& Interfaces, copyright (C American Chemical Society after peer review and technical editing by the publisher. To access the final edited and published work see https://doi.org/10.1021/acsami.8b20751. 


\begin{abstract}
The stabilization of silicene at ambient conditions is essential for its characterization, future processing and device integration. Here, we demonstrate in-situ encapsulation of silicene on Ag(111) by exfoliated few-layer graphene (FLG) flakes, allowing subsequent Raman analysis under ambient conditions. Raman spectroscopy measurements proved that FLG capping serves as an effective passivation, preventing degradation of silicene for up to 48 hours.

The acquired data are consistent with former in-situ Raman measurements, showing two characteristic peaks, located at $216 \mathrm{~cm}^{-1}$ and $515 \mathrm{~cm}^{-1}$. Polarization-dependent measurements allowed to identify the two modes as A and E, demonstrating that the symmetry properties of silicene are unaltered by the capping process.
\end{abstract}




\section{Introduction}

The outstanding electrical properties of silicene are of foremost interest for the development of high performance $2 \mathrm{D}$-material based devices ${ }^{1}$. Silicene combines an ultra-high carrier mobility ${ }^{2-4}$ with the unique opportunity to tune the bandgap by applying an external electric field ${ }^{5,6}$ or by chemical functionalization ${ }^{7,8}$.

Due to the instability of silicene its synthesis requires UHV conditions and a supporting substrate ${ }^{3}$. Silver still remains the most popular choice for growing silicene epitaxially over large areas because of the moderate interaction as well as the low lattice mismatch between the $\operatorname{Ag}(111)$ surface and silicene ${ }^{9,10}$. However, silicene grown on Ag(111) oxidizes when exposed to ambient conditions ${ }^{1,11}$, breaking the hexagonal structure consisting of both $\mathrm{sp}^{2}$ and $\mathrm{sp}^{3}$ hybridized orbitals $^{12,13}$. Therefore, exploring a reliable process for the encapsulation of silicene is fundamental.

An ideal passivation layer has to stabilize silicene as well as enabling the analysis of its structural integrity after subsequent processing steps. The properties of the passivated layer can be investigated by Raman spectroscopy, which has been proven to be an extremely powerful tool for the identification and characterization of $2 \mathrm{D}$ materials ${ }^{14-16}$. At present, a reliable knowledge of the vibrational properties of silicene on $\mathrm{Ag}(111)$ has been gained through a growing number of in-situ Raman experiments ${ }^{15,17,18}$, thus representing a solid model for the identification and analysis of the encapsulated silicene layer. Hence, this non-destructive technique is the method of choice for the identification of silicene in its different phases and for verifying its structural integrity.

Air-stable 2D-materials such as graphene and hexagonal boron nitride (hBN) are considered to be excellent passivation layers due to their inertness ${ }^{19}$, high flexibility ${ }^{20}$ and outstanding impermeability to even the smallest molecules ${ }^{21}$. Moreover, they are optically transparent and nonRaman active in the fingerprint region of silicene and are thus ideal candidates for its stabilization. 
Here we present an encapsulation method based on the in-situ mechanical exfoliation of FLG flakes directly on top of silicene epitaxially grown on $\mathrm{Ag}(111)$.

The FLG is capable of stabilizing silicene up to $48 \mathrm{~h}$ and allows acquiring ex-situ $\mu$-Raman spectra that are in good agreement with in-situ experiments and provide high lateral resolution.

\section{Experimental}

Experiments were performed in an UHV-system at a base pressure of $5 \times 10^{-11} \mathrm{mbar}$. The Ag(111)/mica growth substrate (MaTecK) was cleaned trough several cycles of $\mathrm{Ar}^{+}$ion sputtering ( $1 \mathrm{keV}, 5 \mathrm{~min}$ ), followed by annealing at $800 \mathrm{~K}$ for $20 \mathrm{~min}$. Silicene synthesis was achieved by silicon electron beam evaporation (Specs, EBE-1) with a deposition rate of $\approx 0.02 \mathrm{ML} / \mathrm{min}$ and a substrate temperature of $535 \mathrm{~K}$. The annealing and growth temperatures were controlled by an infrared pyrometer with a precision of $\pm 2 \mathrm{~K}$. The structure and quality of the obtained silicene was verified via LEED measurements.

To passivate silicene, thin graphitic films were prepared by mechanical exfoliation ${ }^{22}$ from natural graphite (HQGraphene) on top of a vacuum compatible polyimide tape (thickness of $\approx 25 \pm 2 \mu \mathrm{m}$ ) with a siliconic adhesive (HB830). The tape was inserted into a specifically designed stamping device located in a dedicated UHV-chamber, directly connected to the growth chamber via a transfer line. The encapsulation process was performed by stamping the previously prepared polyimide tape with graphite flakes onto silicene, at a substrate temperature of $\approx 373 \mathrm{~K}$. The particular stamping device allows a precise control of the encapsulation procedure in order to prevent mechanically-induced damages to the sample.

Ex-situ Raman analysis was performed in a back-scattering geometry using a confocal $\mu$-Raman setup (Alpha300, WITec) with a $532 \mathrm{~nm}(2.33 \mathrm{eV}) \mathrm{Nd}: Y A G$ laser and a spot size of $\approx 720 \mathrm{~nm}$. All 
measurements were performed at ambient conditions and the laser power was chosen to minimize heating effects. For further surface topography analysis, AFM measurements were performed in tapping mode.

\section{Results}

\subsection{FLG capping of silicene}

The Raman measurements revealed large areas of stable passivated silicene under various FLG flakes. However, not all of them were capable of passivating silicene. A large majority of the FLG flakes that encapsulated silicene were found to be between 10 to $15 \mathrm{~nm}$ thick, verified by AFM as presented in Figure 1. This does not necessarily imply that thinner FLG flakes are less suited for the passivation. It rather displays the well-known problem of locating thin 2D materials ${ }^{23,24}$, especially on metallic substrates ${ }^{25}$, where graphene up to a certain amount of layers is essentially invisible. Thicker FLG flakes did not allow a detailed Raman analysis of the underlying substrate. Furthermore, larger flakes showed a higher probability of an intact subjacent silicene layer, since typically no signal of silicene could be detected close to the flake edges. A further issue for the integrity of silicene were the inevitably introduced contaminations from the polyimide tape. Cautious treatment of the FLG flakes and exfoliation tape in cleanroom environment, as well as sufficient bake-out and degassing time in the UHV chamber proved to have significant influence on the yield of capped silicene. 


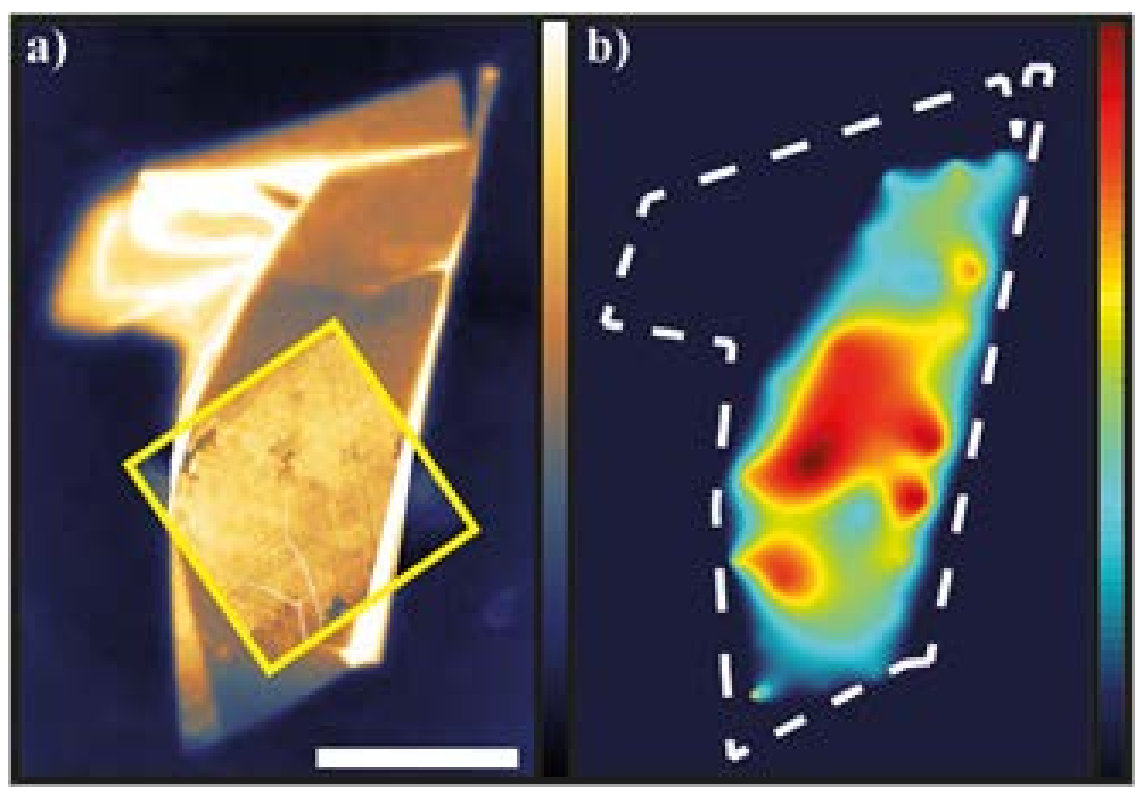

Figure 1. (a) Optical microscope image of a stamped $10 \times 40 \mu \mathrm{m}^{2}$ FLG flake on top of the silicene/Ag(111) surface. The yellow framed area shows a detailed AFM measurement of the capping flake (Lateral scale bar $10 \mu \mathrm{m}$, logarithmic z scale $15 \mathrm{~nm}$ ). (b) Normalized intensity heatmap of the out of plane vibrational silicene Raman mode, interpolated from several dozen Raman point scans. The dashed white line indicates the border of the FLG layer.

Figure 1. (a) illustrates an optical image, including an AFM topography inset, of a $\approx 10 \mathrm{~nm}$ thick and 10x40 $\mu^{2}$ sized FLG flake on top of the Ag(111)/silicene sample after the stamping process. A normalized intensity heatmap of the most prominent silicene related Raman signal can be seen in Figure 1. (b). The intensity of the out of plane vibrational mode was taken as the indication for the presence of silicene.

The encapsulated silicene film showed good stability for up to 48 hours. The inability of permanent stabilization is a problem that also occurs when using graphene as protective capping for other materials such as black phosphorous ${ }^{26,27}$ or metals ${ }^{28,29}$. Capped substrates can experience oxidation at grain boundaries and defects of the protective graphene layer ${ }^{28,29}$. 
For black phosphorous encapsulated by graphene, degradation has been observed from the edges of the graphene crystals ${ }^{26,27}$, which is in good agreement with our experimental observations. Concerning silicene a direct degradation from the edges seems unlikely since oxygen adatoms prefer to accommodate in the surface area rather than at the sides, as the dangling bonds of edge silicon atoms are stabilized by the Ag-substrate ${ }^{30,31}$. Therefore, the degradation is suspected to occur due to an imperfect adhesion of graphene to the substrate, which allows water and oxygen to enter at the interface. Furthermore, there is a clear absence or marked decrease of the silicene Raman signal at macroscopic defects such as wrinkles in the FLG capping layer, indicating a preferential diffusion path for the oxidizing species.

To circumvent this problem further encapsulating materials such as hBN have been suggested. Although, thin hBN capping layers were shown to have similar issues with the long-term stability of subjacent substrates ${ }^{32}$, recent studies showed promising results for multilayered structures ${ }^{33}$. However, our passivation experiments using the same approach with hBN appeared thus far to be incapable of stabilizing silicene.

\subsection{Raman Characterization of Encapsulated Silicene}

Figure 2. (a) shows the Raman spectrum of a FLG-flake encapsulating an epitaxial $4 \times 4$ silicene monolayer, consisting mainly of $4 \times 4$ silicene accompanied by the $(\sqrt{13} \times \sqrt{ } 13) R 13.9^{\circ}$ reconstruction, atop of the $\mathrm{Ag}(111)$ growth substrate. Although the $4 \times 4$ structure was reported to be the most stable one, it coexists with other silicene reconstructions, since their formation energies

are similar ${ }^{34,35}$. In this work we will refer to this mixture of $4 \times 4$ and $(\sqrt{ } 13 \times \sqrt{ } 13) R 13.9^{\circ}$ silicene as silicene only, if not stated otherwise. 


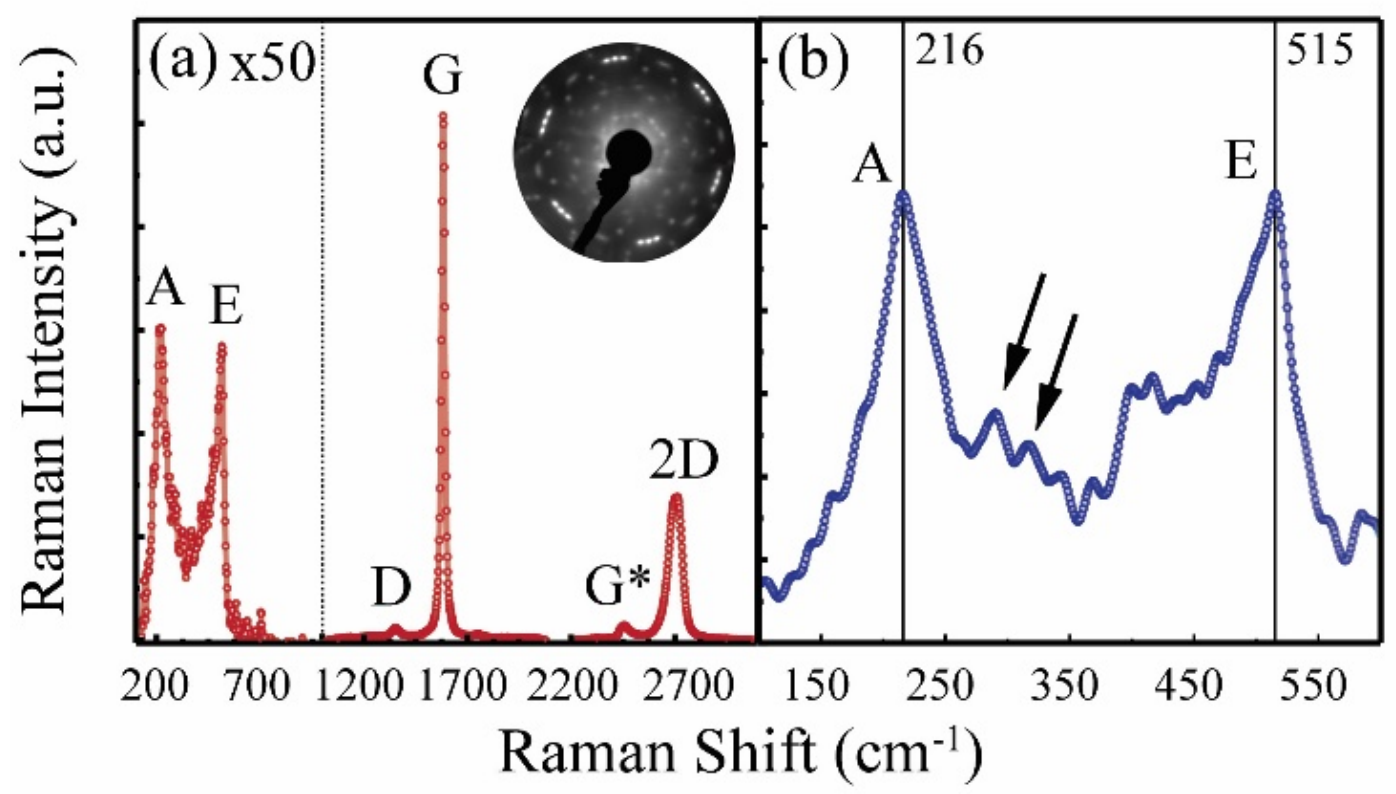

Figure 2. (a) Raman spectrum of the FLG flake encapsulating 1 ML of silicene grown on $\mathrm{Ag}(111)$. Alongside FLG Raman modes, 2 additional peaks assigned to silicene (A and E) can be clearly distinguished in the low frequency region of the spectrum (enlarged 50 times). The small inset shows the silicene LEED pattern. (b) Enlarged view of the low frequency region of the spectrum. Two main modes are clearly visible, located at $216 \mathrm{~cm}^{-1}$ and $515 \mathrm{~cm}^{-1}$. Additional modes can be spotted between $250 \mathrm{~cm}^{-1}$ and $350 \mathrm{~cm}^{-1}$ (indicated by arrows), compatible with literature reported out-of-plane optical phonon (ZO) modes.

The spectrum has been measured at ambient conditions and room temperature, approximately 30 minutes after the sample was removed from the UHV chamber. The Raman signal shows the well-known peaks for exfoliated FLG, located at 1350, 1585, 2450 and $2700 \mathrm{~cm}^{-1}$, labelled, respectively, as D, G, G* and 2D, following literature ${ }^{36,37}$.

Two additional Raman modes can be clearly distinguished at $216 \mathrm{~cm}^{-1}$ and at $515 \mathrm{~cm}^{-1}$. Previous in-situ ${ }^{15,18}$ and ex-situ ${ }^{38}$ Raman studies reliably assigned a similar vibrational signature to the silicene monolayer grown on $\mathrm{Ag}(111)$. 
Figure 2. (b) shows an enlarged view of the fingerprint region of silicene. Raman spectra that are acquired in-situ using macro setups always show a broad peak of a-Si, due to the unavoidable quantity of amorphous silicon grown on unfavorable portions of the Ag substrate. The high signal of the amorphous silicon hinders possible low-intensity Raman modes situated within the 300$500 \mathrm{~cm}^{-1}$ spectral range. The $\mu$-Raman setup used in the present work, instead, is capable of analyzing sub-micrometer areas, allowing highly resolved detection of a-Si-free silicene spectra. The $515 \mathrm{~cm}^{-1}$ phonon mode is reported by both in-situ and ex-situ studies and has been assigned to the $\mathrm{E}_{2 \mathrm{~g}}$ vibration mode for silicene superstructures, related to the longitudinal and transversal in-plane optical (LO and TO) modes ${ }^{15,17,18,38}$. The $216 \mathrm{~cm}^{-1}$ feature, instead, has been assigned to a mode A, related to out-of-plane optical (ZO) phonons. Interestingly, the A mode is reported only by in-situ analysis ${ }^{15,17,18}$. The previous ex-situ Raman study of silicene exploited a different encapsulation method, where the stabilization of the 2D layer is obtained with the deposition of a thin $\mathrm{Al}$ layer that oxidizes preferentially, resulting in an $\mathrm{Al}_{2} \mathrm{O}_{3}$ protecting layer ${ }^{38}$. However, the encapsulating layer is responsible for the presence of a broad peak that likely hinders the detection of certain Raman features.

In addition to the two high intensity peaks, it is also possible to distinguish two small peaks around $\approx 295 \mathrm{~cm}^{-1}$ and $\approx 315 \mathrm{~cm}^{-1}$ (indicated by arrows), which are in agreement with Raman features detected via Tip Enhanced Raman Spectroscopy (TERS) and assigned to ZO vibrational modes ${ }^{17}$. Polarization dependent Raman measurements were conducted in order to confirm unambiguously the identification of silicene and to gain a deeper knowledge on the structure of the capped 2D layer. It is known that the $4 \times 4$ silicene grown on $\mathrm{Ag}(111)$ shows a C6v symmetry, while freestanding silicene belongs to the D3d group. The minority phase $(\sqrt{ } 13 \times \sqrt{ } 13) \mathrm{R} 13.9^{\circ}$, instead, 
belongs to the zero symmetry group $\mathrm{C} 1$. For this reason, the polarization dependent measurements will be discussed only regarding the $4 \times 4$ phase ${ }^{34,39}$.

C6v group possesses three possible phonon symmetries: A, E1 and E2 ${ }^{40}$. The selection rules for this group predict that, in a back-scattering geometry configuration, the A mode is suppressed when the spectrum is collected in crossed polarization configuration $(-\mathrm{Z}(\mathrm{XY}) \mathrm{Z}$ - following Porto notation), while it is visible in parallel polarization configuration $(-\mathrm{Z}(\mathrm{XX}) \mathrm{Z})^{41}$. E1 and E2 modes, instead, are always visible, regardless of the chosen polarization geometry of the Raman setup. For this reason, peaks that belong to one of these two modes will be assigned to a more general $\mathrm{E}$ mode. Figure 3 illustrates the comparison between the spectrum of the capped silicene collected in crossed and parallel polarization configurations.

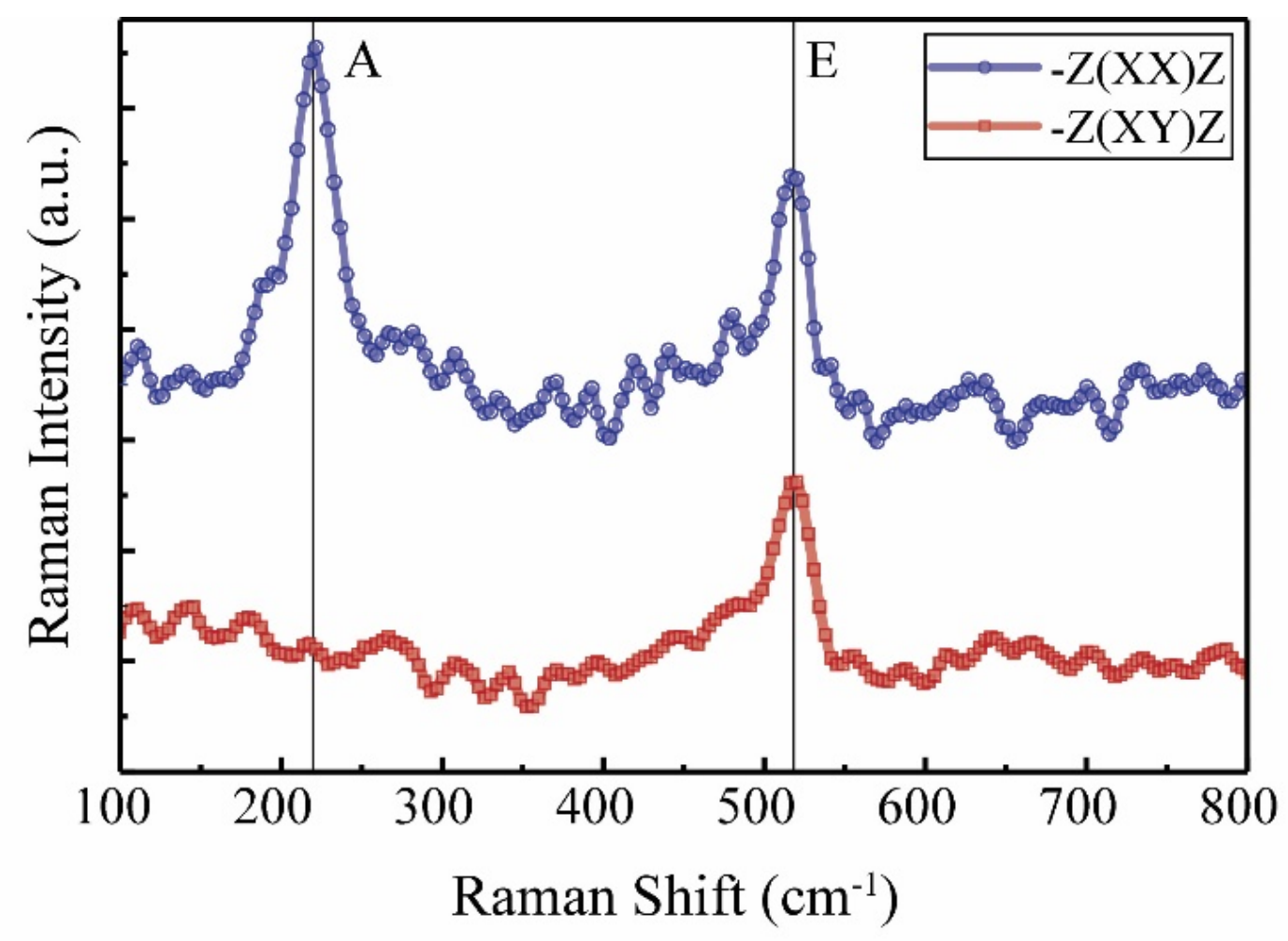

Figure 3. Raman spectrum of silicene collected in back scattering geometry, with parallel polarization configuration $(-\mathrm{Z}(\mathrm{XX}) \mathrm{Z}$ (Porto notation) - blue) and crossed polarization 
configuration (-Z(XY)Z (Porto notation) - red). The Raman mode located at $216 \mathrm{~cm}^{-1}$ is visible only in parallel polarization configuration and can be assigned to the phonon symmetry " $\mathrm{A}$ ". The $515 \mathrm{~cm}^{-1}$ peak is detected in both configurations, thus can be assigned to the "E" symmetry.

Clearly, the peak located at $216 \mathrm{~cm}^{-1}$ vanishes when the spectrum is collected in a crossed polarization geometry, while the one at $515 \mathrm{~cm}^{-1}$ is always visible, in perfect agreement with the results from the in-situ experiment ${ }^{15}$. These observations confirm the assignment of the two peaks to phonon modes A and E. It is important to stress that the appearance of phonon mode A, which is not detected in bulk silicon structures, is a clear indication of the two-dimensionality of the capped material ${ }^{42}$.

It must be noted that the Raman signature of silicene is particularly sensitive to structural changes of the 2D layer. For example, the $\mathrm{E}$ mode location for freestanding silicene is simulated at $570 \mathrm{~cm}^{-1}$ because of the shorter bond length compared to silicene on $\operatorname{Ag}(111)^{43}$. Thus, the actual Raman analysis shows that silicene-FLG interaction is weak enough to preserve the particular structure of silicene. This is crucial for preserving the electrical properties of the silicene layer, as they can be altered by changes in the geometrical structure ${ }^{44,45}$.

The passivating capabilities of the FLG flake were probed by following the time evolution of the encapsulated silicene Raman spectrum while exposing the sample to ambient conditions.

Figure 4 shows the comparison between the spectra acquired 1 hour, 24 hours, 48 hours and 72 hours after the removal of the sample from the UHV chamber. 


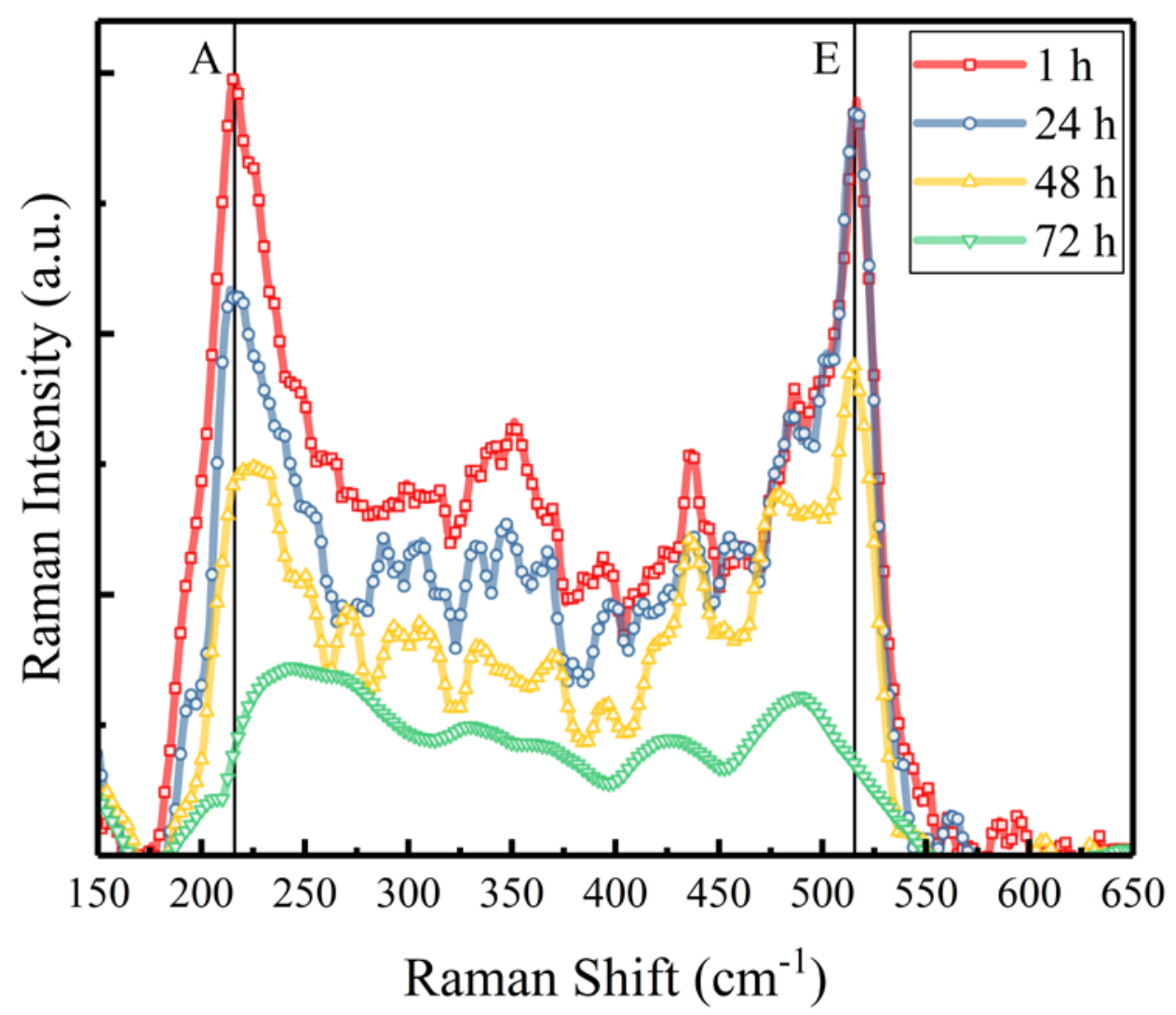

Figure 4. Time evolution of the Raman spectrum of encapsulated silicene exposed to ambient conditions for 1 hour (red), 24 hours (blue), 48 hours (yellow) and 72 hours (green).

The Raman spectra collected after 1 and 24 hours of exposure to ambient conditions look similar, with no visible shift of the two high intensity modes and the same behavior in the spectral region between $250 \mathrm{~cm}^{-1}$ and $500 \mathrm{~cm}^{-1}$. In particular, the intensity of the $\mathrm{E}$ mode is unchanged, while the A mode shows a slightly lower intensity. This difference can be explained by the obvious experimental difficulty to analyze the exactly same spot of the first measurement, thus probing a slightly different silicene area. The $48 \mathrm{~h}$ spectrum clearly shows again the two main $\mathrm{A}$ and $\mathrm{E}$ modes of silicene, without visible Raman shift. The spectrum details in the region between the two main modes are still similar to what was observed after $1 \mathrm{~h}$ of exposure to ambient conditions. A general decrease in intensity, alongside with a rounding of the A mode can be noticed, hinting to 
a start of the degradation process. After $72 \mathrm{~h}$ of exposure to ambient conditions no traces of the presence of silicene can be detected, demonstrating a complete degradation of the 2D layer. It must be noted that all Raman spectra acquired outside of an encapsulation area resemble the green spectrum of Figure 4, even few minutes after the removal of the sample from the UHV chamber, thus proving the capping capabilities of the FLG layer. Finally, it must be taken into consideration that the measurement process can locally heat the FLG flake ${ }^{46}$. For this reason, a repeated measurement could speed up the degradation process, as the contact between silicene and the capping flake deteriorates due to thermal shrinking and consequent expansion of the FLG layer ${ }^{47}$. To prove these assumptions, we examined a sample with two FLG flakes successfully passivating silicene. One flake was analyzed approximately after $1 \mathrm{~h}$ of exposure to ambient conditions, while the second flake was measured only once after $36 \mathrm{~h}$. As provided in the supplementary information, the two spectra show the same Raman intensities. Thus, we conclude that the Raman measurement itself accelerates the degradation.

The passivation method was applied to study the Raman spectra of samples characterized by different Si coverages $(\Theta)$. By varying the evaporation time, it was possible to obtain samples with $\Theta$ of $\approx 0.7 \mathrm{ML}, \approx 1 \mathrm{ML}, \approx 1.3 \mathrm{ML}, \approx 2 \mathrm{ML}$ and $\approx 2.7 \mathrm{ML}$. Each sample was then passivated following the method described above and the Raman spectra were acquired ex-situ within 1 hour after the removal from the UHV chamber.

Figure 5 shows the comparison between the normalized spectra of the respective samples. The samples with $\Theta \leq 1$ ML show a Raman spectrum mainly dominated by the A and E phonon modes, as previously discussed. The respective LEED patterns in the insets show the typical reconstruction for monolayer silicene grown on $\mathrm{Ag}(111)$ at $530 \mathrm{~K}$ with a prominent signal from the $4 \mathrm{x} 4$ phase 
mixed with a low signal for the minority $(\sqrt{ } 13 \times \sqrt{ } 13) \mathrm{R} 13.9^{\circ}$ reconstruction $^{35}$. The LEED pattern for the sample with $\Theta \approx 1 \mathrm{ML}$ is clearly more intense than the pattern observed for the sample with $\Theta \approx 0.7 \mathrm{ML}$, due to the presence of larger silicene domains.

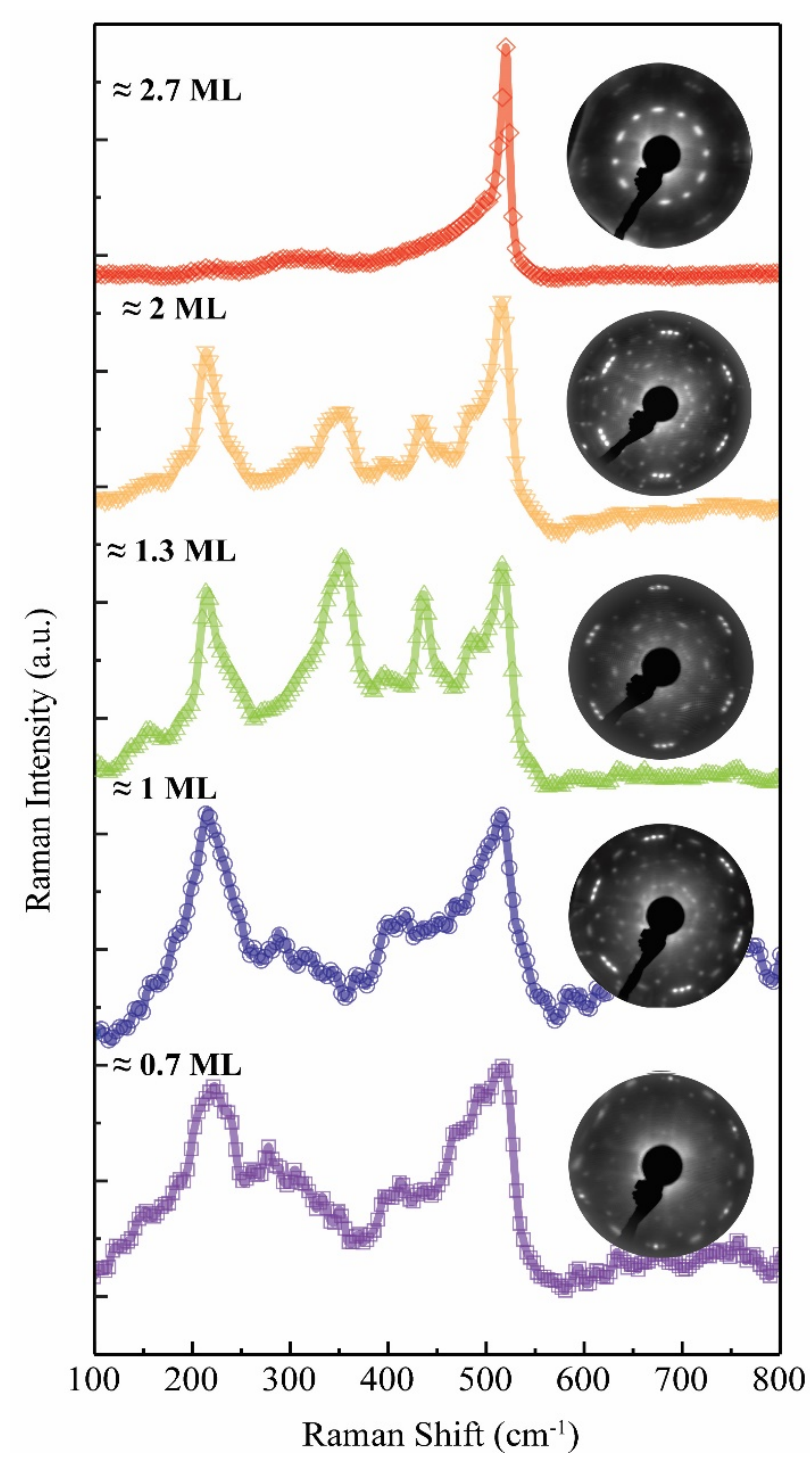

Figure 5. Normalized Raman spectra and LEED pattern of samples characterized by different silicon coverages $(\Theta)$. From bottom to top: spectrum of $\approx 0.7$ ML silicene (purple) characterized by the 2 main modes $\mathrm{A}$ and $\mathrm{E}$ of monolayer silicene, with respective LEED picture showing a $4 \mathrm{x} 4$ silicene reconstruction with a minority $(\sqrt{ } 13 \times \sqrt{ } 13) \mathrm{R} 13.9^{\circ}$ phase; spectrum of $\approx 1$ ML silicene (blue), with respective LEED picture showing an high intensity signal from a $4 \times 4$ silicene 
reconstruction with a minority $(\sqrt{ } 13 \times \sqrt{ } 13) \mathrm{R} 13.9^{\circ}$ phase; spectrum of $\approx 1.3$ ML silicene (green) characterized by the appearance of additional peaks between the A and E mode, with respective LEED picture showing mixture of $4 \times 4$ and $(\sqrt{ } 13 \times \sqrt{ } 13) R 13.9^{\circ}$ reconstruction, due to the monolayer regions, and $\frac{4}{\sqrt{3}} \times \frac{4}{\sqrt{3}} \mathrm{R} 30^{\circ}$, attributed to the presence of regions where $\Theta$ exceeds $1 \mathrm{ML}$; spectrum of $\approx 2$ ML silicene (orange) characterized by a lower intensity of the additional peaks between the $A$ and $E$ mode, with respective LEED picture showing mixture of $4 \times 44,(\sqrt{ } 13 x$

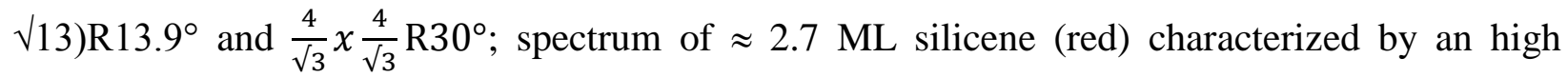
intensity peak located at $\approx 520 \mathrm{~cm}^{-1}$, with respective LEED picture showing an intense signal from the $\frac{4}{\sqrt{3}} \times \frac{4}{\sqrt{3}} \mathrm{R} 30^{\circ}$ phase, together with a faint signal from the $4 \times 4$ and $(\sqrt{ } 13 \times \sqrt{ } 13) \mathrm{R} 13.9^{\circ}$ reconstructions.

When $\Theta$ exceeds 1 ML the Raman spectrum clearly changes. While A and E modes are still visible, two intense and sharp peaks appear around $\approx 350 \mathrm{~cm}^{-1}$ and $\approx 450 \mathrm{~cm}^{-1}$. Similar Raman features were previously reported by an in-situ Raman study for multilayer silicene samples ${ }^{18}$. These additional Raman peaks were tentatively assigned to edge-activated phonon modes, similar to what can be observed in graphene flakes, in which defects are responsible for double-resonating Raman scattering processes ${ }^{48}$. The LEED pattern changes, now showing a mixture of $4 x 4$ and $(\sqrt{ } 13 x$ $\sqrt{13) R 13.9^{\circ}}$ silicene, due to the monolayer regions, and $\frac{4}{\sqrt{3}} \times \frac{4}{\sqrt{3}} \mathrm{R} 30^{\circ}$, attributed to the presence of regions where $\Theta$ exceeds $1 \mathrm{ML}^{49,50}$. When $\Theta$ is further increased towards 2 ML, the additional Raman peaks are still visible but their intensity lowers with respect to the intensity of the A and E modes. The LEED pattern still shows a mixed reconstruction of $4 \times 4,(\sqrt{ } 13 \times \sqrt{ } 13) R 13.9^{\circ}$ and $\frac{4}{\sqrt{3}} \times \frac{4}{\sqrt{3}} \mathrm{R} 30^{\circ}$ phases. It can be assumed that the completion of the second layer leads to a reduction in the density of domain edges. This observation supports the identification of the new Raman 
modes as defect-activated. It must be noted, however, that the TERS experiment clearly shows that the edges of the first silicene layer are not activating any of these additional Raman modes ${ }^{17}$, thus being related only to the edges of the topmost layer. As the existence of multilayer silicene is still under debate ${ }^{50-52}$, one cannot exclude the possibility that these additional Raman modes are, instead, appearing due to a change in the crystalline structure of the analyzed layer, promoted by the higher density of Si atoms.

The sample with $\Theta \approx 2.7$ ML is characterized by a drastically different Raman spectrum and LEED pattern. The spectrum shows a single Raman peak, centered at $\approx 520 \mathrm{~cm}^{-1}$, with a much higher intensity than the silicene A and E peaks (50x more intense in the non-normalized data). Remarkably, the same spectrum can be detected also analyzing areas that are outside of the capping flake, demonstrating the great air stability of the layer. The LEED pattern changed as well. The pattern is now prevalently $\frac{4}{\sqrt{3}} \times \frac{4}{\sqrt{3}} \mathrm{R} 30^{\circ}$, mixed with a very low-intensity $4 \times 4$ and $(\sqrt{ } 13 \mathrm{x}$ $\sqrt{13) R} 13.9^{\circ}$ pattern. It has been shown that this peculiar LEED pattern could be related to a surface reconstruction of Ag atoms that floated on top of the Si layer during the evaporation process $s^{50,53}$. In-situ Raman studies of multilayer silicene have reported a slightly different spectrum than the one shown here, characterized by an intense peak located at $\approx 523 \mathrm{~cm}^{-1}$ and a broad band located between $430 \mathrm{~cm}^{-1}$ and $500 \mathrm{~cm}^{-154}$. The $\approx 3 \mathrm{~cm}^{-1}$ shift of the main peak can be attributed to the presence of stacking faults that cause the in-plane lattice constant to be smaller ${ }^{53}$. The increased presence of stacking faults in the mentioned in-situ study can be explained by the choice of a particularly low growth temperature $(470 \mathrm{~K})$, with respect to the one of the present work ${ }^{52}$. The broad band, instead, can be assigned to the presence of amorphous silicon. 


\section{Conclusion}

In conclusion, we present a novel method for the stabilization of silicene on $\mathrm{Ag}(111)$ up to $48 \mathrm{~h}$. In UHV, an FLG flake is mechanically exfoliated atop of epitaxially-grown silicene, forming an encapsulation layer. This allows to perform high quality, spatially resolved, ex-situ Raman analysis of the silicene layer thanks to the Raman inactivity of the FLG flake in the spectral region of interest. The observed vibrational signature matches with the in-situ Raman spectra reported in literature, proving that the encapsulation layer does not alter the overall structure of silicene. The method has been applied to study the Raman signature of silicene samples characterized by a Si coverage spanning between $0.7 \mathrm{ML}$ and $2.7 \mathrm{ML}$. The present work is also confirming the compatibility between graphene and silicene, representing a step forward towards the integration of silicene into 2D heterostructures.

\section{AUTHOR INFORMATION}

\section{Alois Lugstein}

Institute of Solid State Electronics, Technische Universität Wien, Gußhausstraße 25-25a, 1040

Vienna, Austria

\section{Author Contributions}

Viktoria Ritter, Jakob Genser and Daniele Nazzari contributed equally to this work.

\section{ACKNOWLEDGMENT}

This work was funded by the Fonds zur Förderung der Wissenschaftlichen Forschung (FWF), Austria (project P29244-N27). We thank the center for micro- and nanostructures (ZMNS) at the 
TU Vienna for access to the cleanroom facilities. We would like to thank Harold J. W. Zandvliet from the University of Twente for support in the synthesis of silicene and Alessandro Molle from the Institute for Microelectronics and Microsystems for the fruitful discussions on the encapsulation process.

\section{SUPPORTING INFORMATION}

Raman spectra showing the influence of the measurement process on the degradation of silicene under a FLG flake.

\section{REFERENCES}

(1) Tao, L.; Cinquanta, E.; Chiappe, D.; Grazianetti, C.; Fanciulli, M.; Dubey, M.; Molle, A.; Akinwande, D. Silicene Field-Effect Transistors Operating at Room Temperature. Nat. Nanotechnol. 2015, 10 (3), 227-231.

(2) Le Lay, G. Silicene Transistors. Nat. Nanotechnol. 2015, 10 (3), 202-203.

(3) Zhao, J.; Liu, H.; Yu, Z.; Quhe, R.; Zhou, S.; Wang, Y.; Liu, C. C.; Zhong, H.; Han, N.; Lu, J.; Yao, Y.; Wu, K. Rise of Silicene: A Competitive 2D Material. Prog. Mater. Sci. 2016, 83, 24-151.

(4) Lew Yan Voon, L. C. Electronic Structure of Silicene. Chinese Phys. B 2015, 24 (8), 087309.

(5) Drummond, N. D.; Zólyomi, V.; Fal’ko, V. I. Electrically Tunable Band Gap in Silicene. Phys. Rev. B 2012, 85 (7), 075423.

(6) Ni, Z.; Liu, Q.; Tang, K.; Zheng, J.; Zhou, J.; Qin, R.; Gao, Z.; Yu, D.; Lu, J. Tunable Bandgap in Silicene and Germanene. Nano Lett. 2012, 12 (1), 113-118.

(7) Gao, N.; Zheng, W. T.; Jiang, Q. Density Functional Theory Calculations for Two- 
Dimensional Silicene with Halogen Functionalization. Phys. Chem. Chem. Phys. 2012, 14 (1), 257-261.

(8) Dávila, M. E.; Marele, A.; De Padova, P.; Montero, I.; Hennies, F.; Pietzsch, A.; Shariati, M. N.; Gómez-Rodríguez, J. M.; Le Lay, G. Comparative Structural and Electronic Studies of Hydrogen Interaction with Isolated versus Ordered Silicon Nanoribbons Grown on Ag(110). Nanotechnology 2012, 23 (38), 385703.

(9) Vogt, P.; De Padova, P.; Quaresima, C.; Avila, J.; Frantzeskakis, E.; Asensio, M. C.; Resta, A.; Ealet, B.; Le Lay, G. Silicene: Compelling Experimental Evidence for Graphenelike Two-Dimensional Silicon. Phys. Rev. Lett. 2012, 108 (15), 155501.

(10) Chun-Liang, L.; Ryuichi, A.; Maki, K.; Noriaki, T. Comparison of Electronic Structure between Monolayer Silicenes on Ag (111). Chinese Phys. B 2015, 24 (8), 087307.

(11) Friedlein, R.; Van Bui, H.; Wiggers, F. B.; Yamada-Takamura, Y.; Kovalgin, A. Y.; de Jong, M. P. Interaction of Epitaxial Silicene with Overlayers Formed by Exposure to Al Atoms and O2 Molecules. J. Chem. Phys. 2014, 140 (20), 204705.

(12) Cahangirov, S.; Sahin, H.; Le Lay, G.; Rubio, A. Freestanding Silicene. In Introduction to the Physics of Silicene and other 2D Materials 2017.

(13) Cinquanta, E.; Scalise, E.; Chiappe, D.; Grazianetti, C.; van den Broek, B.; Houssa, M.; Fanciulli, M.; Molle, A. Getting through the Nature of Silicene: An sp2-sp3 TwoDimensional Silicon Nanosheet. J. Phys. Chem. C 2013, 117 (32), 16719-16724.

(14) Jose, D.; Nijamudheen, A.; Datta, A. Tip Enhanced Raman Spectroscopy (TERS) as a Probe for the Buckling Distortion in Silicene. Phys. Chem. Chem. Phys. 2013, 15 (22), 8700.

(15) Solonenko, D.; Gordan, O. D.; Lay, G. Le; Şahin, H.; Cahangirov, S.; Zahn, D. R. T.; Vogt, P. 2D Vibrational Properties of Epitaxial Silicene on Ag(111). 2D Mater. 2016, 4 (1), 
015008.

(16) Yan, J.-A.; Stein, R.; Schaefer, D. M.; Wang, X.-Q.; Chou, M. Y. Electron-Phonon Coupling in Two-Dimensional Silicene and Germanene. Phys. Rev. B 2013, 88 (12), 121403.

(17) Sheng, S.; Wu, J.; Cong, X.; Li, W.; Gou, J.; Zhong, Q.; Cheng, P.; Tan, P.; Chen, L.; Wu, K. Vibrational Properties of a Monolayer Silicene Sheet Studied by Tip-Enhanced Raman Spectroscopy. Phys. Rev. Lett. 2017, 119 (19), 196803.

(18) Zhuang, J.; Xu, X.; Du, Y.; Wu, K.; Chen, L.; Hao, W.; Wang, J.; Yeoh, W. K.; Wang, X.; Dou, S. X. Investigation of Electron-Phonon Coupling in Epitaxial Silicene by in Situ Raman Spectroscopy. Phys. Rev. B 2015, 91 (16), 161409.

(19) Meric, I.; Dean, C. R.; Petrone, N.; Wang, L.; Hone, J.; Kim, P.; Shepard, K. L. Graphene Field-Effect Transistors Based on Boron-Nitride Dielectrics. Proc. IEEE 2013, 101 (7), 1609-1619.

(20) Akinwande, D.; Petrone, N.; Hone, J. Two-Dimensional Flexible Nanoelectronics. Nat. Commun. 2014, 5 (1), 5678.

(21) Bunch, J. S.; Verbridge, S. S.; Alden, J. S.; van der Zande, A. M.; Parpia, J. M.; Craighead, H. G.; McEuen, P. L. Impermeable Atomic Membranes from Graphene Sheets. Nano Lett. 2008, 8 (8), 2458-2462.

(22) Novoselov, K. S.; Geim, A. K.; Morozov, S. V.; Jiang, D. A.; Zhang, Y.; Dubonos, S. V.; Grigorieva, I. V.; Firsov, A. A. Electric Field Effect in Atomically Thin Carbon Films. Science 2004, 306 (5696), 666-669.

(23) Blake, P.; Hill, E. W.; Castro Neto, A. H.; Novoselov, K. S.; Jiang, D.; Yang, R.; Booth, T. J.; Geim, A. K. Making Graphene Visible. Appl. Phys. Lett. 2007, 91 (6), 063124. 
(24) Gorbachev, R. V.; Riaz, I.; Nair, R. R.; Jalil, R.; Britnell, L.; Belle, B. D.; Hill, E. W.; Novoselov, K. S.; Watanabe, K.; Taniguchi, T.; Geim, A. K.; Blake, P. Hunting for Monolayer Boron Nitride: Optical and Raman Signatures. Small 2011, 7 (4), 465-468.

(25) Velický, M.; Hendren, W. R.; Donnelly, G. E.; Katzen, J. M.; Bowman, R. M.; Huang, F. Optimising the Visibility of Graphene and Graphene Oxide on Gold with Multilayer Heterostructures. Nanotechnology 2018, 29 (27), 275205.

(26) Kim, J.; Baek, S. K.; Kim, K. S.; Chang, Y. J.; Choi, E. J. Long-Term Stability Study of Graphene-Passivated Black Phosphorus under Air Exposure. Curr. Appl. Phys. 2016, 16 (2), 165-169.

(27) Doganov, R. A.; O’Farrell, E. C. T.; Koenig, S. P.; Yeo, Y.; Ziletti, A.; Carvalho, A.; Campbell, D. K.; Coker, D. F.; Watanabe, K.; Taniguchi, T.; Neto, A. H. C.; Özyilmaz, B. Transport Properties of Pristine Few-Layer Black Phosphorus by van Der Waals Passivation in an Inert Atmosphere. Nat. Commun. 2015, 6 (1), 6647.

(28) Dedkov, Y. S.; Fonin, M.; Laubschat, C. A Possible Source of Spin-Polarized Electrons: The Inert Graphene/Ni(111) System. Appl. Phys. Lett. 2008, 92 (5), 052506.

(29) Chen, S.; Brown, L.; Levendorf, M.; Cai, W.; Ju, S.-Y.; Edgeworth, J.; Li, X.; Magnuson, C. W.; Velamakanni, A.; Piner, R. D.; Kang, J.; Park, J.; Ruoff, R. S. Oxidation Resistance of Graphene-Coated Cu and Cu/Ni Alloy. ACS Nano 2011, 5 (2), 1321-1327.

(30) Du, Y.; Zhuang, J.; Liu, H.; Xu, X.; Eilers, S.; Wu, K.; Cheng, P.; Zhao, J.; Pi, X.; See, K. W.; Peleckis, G.; Wang, X.; Dou, S. X. Tuning the Band Gap in Silicene by Oxidation. ACS Nano 2014, 8 (10), 10019-10025.

(31) Ali, M.; Ni, Z.; Cottenier, S.; Liu, Y.; Pi, X.; Yang, D. Formation, Structures and Electronic Properties of Silicene Oxides on Ag(111). J. Mater. Sci. Technol. 2017, 33 (7), 751-757. 
(32) Island, J. O.; Steele, G. A.; Zant, H. S. J. van der; Castellanos-Gomez, A. Environmental Instability of Few-Layer Black Phosphorus. 2D Mater. 2015, 2 (1), 011002.

(33) Li, L.; Kim, J.; Jin, C.; Ye, G. J.; Qiu, D. Y.; da Jornada, F. H.; Shi, Z.; Chen, L.; Zhang, Z.; Yang, F.; Watanabe, K.; Taniguchi, T.; Ren, W.; Louie, S. G.; Chen, X. H.; Zhang, Y.; Wang, F. Direct Observation of the Layer-Dependent Electronic Structure in Phosphorene. Nat. Nanotechnol. 2016, 12 (1), 21-25.

(34) Pflugradt, P.; Matthes, L.; Bechstedt, F. Silicene-Derived Phases on Ag(111) Substrate versus Coverage: Ab Initio Studies. Phys. Rev. B 2014, 89 (3), 035403.

(35) Moras, P.; Mentes, T. O.; Sheverdyaeva, P. M.; Locatelli, A.; Carbone, C. Coexistence of Multiple Silicene Phases in Silicon Grown on Ag(1 1 1). J. Phys. Condens. Matter 2014, 26 (18), 185001.

(36) Ferrari, A. C.; Meyer, J. C.; Scardaci, V.; Casiraghi, C.; Lazzeri, M.; Mauri, F.; Piscanec, S.; Jiang, D.; Novoselov, K. S.; Roth, S.; Geim, A. K. Raman Spectrum of Graphene and Graphene Layers. Phys. Rev. Lett. 2006, 97 (18), 187401.

(37) Malard, L. M.; Pimenta, M. A.; Dresselhaus, G.; Dresselhaus, M. S. Raman Spectroscopy in Graphene. Phys. Rep. 2009, 473 (5-6), 51-87.

(38) Molle, A.; Grazianetti, C.; Chiappe, D.; Cinquanta, E.; Cianci, E.; Tallarida, G.; Fanciulli, M. Hindering the Oxidation of Silicene with Non-Reactive Encapsulation. Adv. Funct. Mater. 2013, 23 (35), 4340-4344.

(39) Ribeiro-Soares, J.; Almeida, R. M.; Cançado, L. G.; Dresselhaus, M. S.; Jorio, A. Group Theory for Structural Analysis and Lattice Vibrations in Phosphorene Systems. Phys. Rev. B 2015, 91 (20), 205421.

(40) Dresselhaus, M. S.; Dresselhaus, G.; Jorio, A. (Ado). Group Theory: Application to the 
Physics of Condensed Matter Springer-Verlag 2008.

(41) Harris, D. C.; Bertolucci, M. D. Symmetry and Spectroscopy: An Introduction to Vibrational and Electronic Spectroscopy Dover Publications 1989.

(42) Richter, H.; Wang, Z. P.; Ley, L. The One Phonon Raman Spectrum in Microcrystalline Silicon. Solid State Commun. 1981, 39 (5), 625-629.

(43) Houssa, M.; Pourtois, G.; Afanas’ev, V. V.; Stesmans, A. Can Silicon Behave like Graphene? A First-Principles Study. Appl. Phys. Lett. 2010, 97 (11), 112106.

(44) Lin, C.-L.; Arafune, R.; Kawahara, K.; Kanno, M.; Tsukahara, N.; Minamitani, E.; Kim, Y.; Kawai, M.; Takagi, N. Substrate-Induced Symmetry Breaking in Silicene. Phys. Rev. Lett. 2013, 110 (7), 076801.

(45) Liu, C.-C.; Feng, W.; Yao, Y. Quantum Spin Hall Effect in Silicene and Two-Dimensional Germanium. Phys. Rev. Lett. 2011, 107 (7), 076802.

(46) Everall, N. .; Lumsdon, J.; Christopher, D. . The Effect of Laser-Induced Heating upon the Vibrational Raman Spectra of Graphites and Carbon Fibres. Carbon N. Y. 1991, 29 (2), 133-137.

(47) Yoon, D.; Son, Y.-W.; Cheong, H. Negative Thermal Expansion Coefficient of Graphene Measured by Raman Spectroscopy. Nano Lett. 2011, 11 (8), 3227-3231.

(48) Basko, D. M. Theory of Resonant Multiphonon Raman Scattering in Graphene. Phys. Rev. B 2008, 78 (12), 125418.

(49) Salomon, E.; El Ajjouri, R.; Le Lay, G.; Angot, T. Growth and Structural Properties of Silicene at Multilayer Coverage. J. Phys. Condens. Matter 2014, 26 (18), 185003.

(50) Shirai, T.; Shirasawa, T.; Hirahara, T.; Fukui, N.; Takahashi, T.; Hasegawa, S. Structure Determination of Multilayer Silicene Grown on $\mathrm{Ag}(111)$ Films by Electron Diffraction: 
Evidence for Ag Segregation at the Surface. Phys. Rev. B 2014, 89 (24), 241403.

(51) Kamal, C.; Chakrabarti, A.; Banerjee, A.; Deb, S. K. Silicene beyond Mono-Layersdifferent Stacking Configurations and Their Properties. J. Phys. Condens. Matter 2013, 25 (8), 085508.

(52) De Padova, P.; Generosi, A.; Paci, B.; Ottaviani, C.; Quaresima, C.; Olivieri, B.; Salomon, E.; Angot, T.; Le Lay, G. Multilayer Silicene: Clear Evidence. 2D Mater. 2016, 3 (3), 031011.

(53) Curcella, A.; Bernard, R.; Borensztein, Y.; Lazzeri, M.; Resta, A.; Garreau, Y.; Prévot, G. Multilayer Silicene: Clear Evidence of Ag-Terminated Bulk Silicon. 2D Mater. 2017, 4 (2), 025067.

(54) De Padova, P.; Ottaviani, C.; Quaresima, C.; Olivieri, B.; Imperatori, P.; Salomon, E.; Angot, T.; Quagliano, L.; Romano, C.; Vona, A.; Muniz-Miranda, M.; Generosi, A.; Paci, B.; Le Lay, G. 24 h Stability of Thick Multilayer Silicene in Air. 2D Mater. 2014, 1 (2), 021003. 


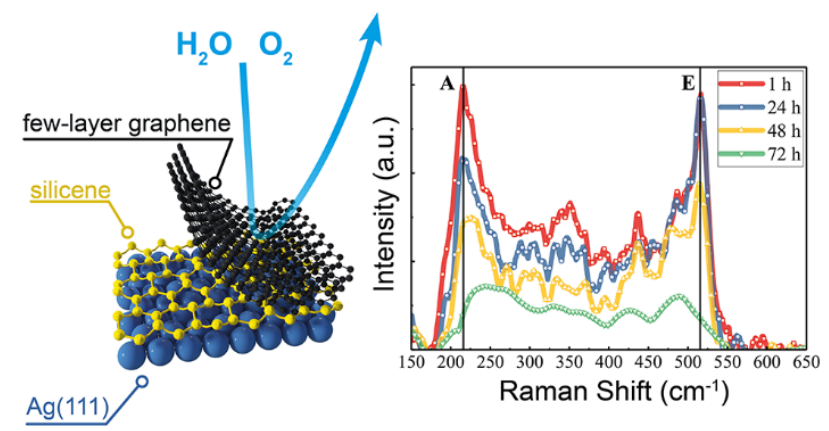

Figure 6. For Table of Contents Only 